\title{
Prevalência de sintomas depressivos em pacientes de serviços ambulatoriais de clínica médica
}

\author{
Prevalence of depressive symptoms in patients \\ of medical ambulatory services \\ Breno Guedes de Melo? \\ https://orcid.org/0000-0001-7260-0361 \\ Antonio Eduardo Silva Junior ${ }^{1}$ \\ https://orid.org//0000-0001-5812-5410 \\ Marina Travassos Lopes ${ }^{1}$ \\ https://orcid.org/0000-0002-4126-1237 \\ Luara Gomes da Silva' \\ https://orcid.org/0000-0002-6850-1042 \\ Roberto Mendes dos Santos' \\ https://orcid.org/0000-0003-0829-5483
}

\section{RESUMO}

Objetivo: Estimar a prevalência de sintomas depressivos em pacientes de ambulatórios de clínica médica no Hospital Universitário Lauro Wanderley, em 2018. Métodos: Aplicação de um questionário sociodemográfico e da escala de depressão de Hamilton em um período de dois meses; 101 pacientes foram devidamente entrevistados. Resultados: Observou-se que $61,4 \%$ da amostra classifica-se entre ligeiramente e gravemente deprimida, sendo 78\% dos pacientes da reumatologia, 63\% da cardiologia e 48\% da endocrinologia. Dos pacientes abordados, 34,6\% praticam atividade física pelo menos três vezes por semana, 53,5\% fazem ou já fizeram uso de medicação para dormir, 25,7\% já fizeram tratamento psiquiátrico e 4,9\% o fazem atualmente, em contrapartida 29,7\% fazem uso de algum psicotrópico. Dos psicotrópicos, o mais utilizado foi o diazepam (16,7\%). Verificaram-se as seguintes associações: pacientes ligeiramente a gravemente deprimidos não praticavam atividades físicas, já faziam tratamento de saúde e tinham histórico de tratamento psiquiátrico; a reumatologia apresentou mais pacientes com algum grau de depressão. Dos 27 que disseram ter sono ruim, $78 \%$ usavam medicamento para dormir e 63\%, psicotrópicos; $60 \%$ não praticavam atividade física e $81 \%$ eram ligeiramente a gravemente deprimidos. Sintomas somáticos foram os mais relatados na escala de Hamilton. Conclusão: Existe alta prevalência de sintomas depressivos em pacientes dos ambulatórios de clínica médica, com destaque para as doenças cardiovasculares, endocrinológicas e reumatológicas. Percebe-se que houve grande destaque para os sintomas somáticos, o que pode explicar a grande quantidade de entrevistados que não são acompanhados por psiquiatra e tratados apenas com sintomáticos.

1 Universidade Federal da Paraíba (UFPB), João Pessoa, PB, Brasil.

Recebido em

18/Out/2019

Aprovado em

$27 / \mathrm{Nov} / 2019$
Endereço para correspondência: Breno MeloCampus I - Lot. Cidade Universitária 58051-900 - PB, João Pessoa, Brasil E-mail: brenogmelo@hotmail.com 


\section{Keywords}

Depression, ambulatory

patient, prevention.

\section{ABSTRACT}

Objective: Estimate the prevalence of depressive symptoms in ambulatory of medical clinics at Lauro Wanderley University Hospital in 2018. Methods: Application of a sociodemographic questionnaire and the Hamilton Depression Rating Scale in a 2-month period, where 101 patients were interviewed. Results: It was observed that $61.4 \%$ of the sample is classified as mildly and severely depressed, with $78 \%$ of rheumatology patients, $63 \%$ of cardiology and $48 \%$ of endocrinology. Of the patients treated $34.6 \%$ practice physical activity at least three times a week, 53.5\% use or had used sleeping medication, 25.7\% have psychiatric treatment and $4.9 \%$ currently do, in contrast $29.7 \%$ use some psychotropic. Of the psychotropics, the most used was Diazepam (16.7\%). The following associations were found: slightly depressed patients did not practice physical activities, were already receiving health treatment and had a history of psychiatric treatment; rheumatology showed more patients with some degree of depression. Of the 27 who reported poor sleep, 78\% used sleeping medication and 63\% psychotropic, $60 \%$ did not practice physical activity and $81 \%$ were slightly depressed. Somatic symptoms were the most reported on the Hamilton scale. Conclusion: There is a high prevalence of depressive symptoms in patients of medical ambulatory services, especially cardiovascular, endocrinological and rheumatologic diseases. It is noticed that there was a great emphasis on somatic symptoms, which may explain the large number of respondents who are not accompanied by psychiatrist and treated only with symptomatic.

\section{INTRODUÇÃO}

Transtornos mentais são patologias frequentes na população brasileira, com prevalência, segundo a CID-10 (Classificação Internacional de Doenças), de aproximadamente 45,9\% durante a vida. Uma pesquisa feita em um ambulatório geral da Universidade Estadual do Rio de Janeiro, composta por 2.792 pacientes, mostrou morbidade psiquiátrica de $38 \%$, e os diagnósticos mais frequentes foram: transtorno de ansiedade generalizada (23\%), episódio depressivo (16\%) transtorno de somatização (9\%), dependência ou uso nocivo de álcool (6\%), distimia (2,5\%) e hipocondria (1\%)'. O São Paulo Megacity Mental Health Survey, por sua vez, trouxe o transtorno depressivo maior como a desordem mais prevalente, o que traz à tona a importância da investigação desse transtorno a fim de basear futuras intervenções populacionais².

A depressão, de acordo com as últimas estimativas da Organização Mundial da Saúde ${ }^{3}$, afeta mais de 300 milhões de pessoas no mundo. Segundo a $5^{a}$ edição do Manual Diagnóstico e Estatístico de Transtornos Mentais (DSM-5), um indivíduo com transtorno depressivo maior deve apresentar, em um tempo mínimo de duas semanas, ao menos cinco de nove sintomas listados no manual, que envolvem desde humor deprimido, diminuição do interesse ou prazer nas atividades cotidianas até ideação suicida ou tentativa de suicídio, e para fechar o diagnóstico é necessário que pelo menos um dos dois primeiros sintomas supracitados estejam presentes.

Segundo Humes et al. ${ }^{4}$, existem evidências epidemiológicas da associação entre transtornos mentais e doenças clínicas e vice-versa, como: $50 \%$ dos pacientes possuem diagnósticos clínicos e psiquiátricos, ou 25\% a 40\% dos pacientes na atenção primária têm transtornos mentais ou, ainda, 50\% dos pacientes com síndromes psiquiátricas crônicas apresentam condições clínicas significativas.

Essa associação entre depressão e doenças clínicas não é unilateral, sendo estas influenciadas mutuamente: a depressão pode ser uma complicação da doença clínica ou de seu tratamento, mas também a complicação clínica pode ser vista como uma consequência dos sintomas depressivos ou, até mesmo, de um ou mais fatores etiológicos comuns a ambas ${ }^{5}$.

De acordo com Furlanetto e Brasil ${ }^{6}$, estudos recentes têm mostrado de forma consistente que indivíduos com doenças clínicas que estão deprimidos apresentam menor adesão aos tratamentos propostos, comportamentos desfavoráveis de autocuidado, maior prejuízo funcional, diminuição da qualidade de vida, aumento nos custos e pior prognóstico, com maior morbidade e mortalidade, corroborando mais uma vez as consequências da associação entre depressão e doenças clínicas.

Outros dados da literatura sugerem que uma considerável proporção de pacientes passará despercebida ou será diagnosticada de forma inadequada e, em alguns casos, será tratada incorretamente por seus médicos. Assim, muitos pacientes clínicos podem estar em sofrimento mental concomitante e precisam ser atendidos e manejados com mais cautela e responsabilidade 4 .

Diante da problemática em tela, é notória a importância de estudos epidemiológicos e estatísticos sobre depressão, a fim de maior entendimento da população dos serviços de saúde, permitindo a criação de estratégias de cuidado mais eficazes. O objetivo deste trabalho foi pesquisar as princi- 
pais características clínicas e sociodemográficas associadas a sintomas depressivos nos pacientes atendidos em ambulatórios de três especialidades de clínica médica do Hospital Universitário Lauro Wanderley, propondo uma alternativa para identificar mais facilmente pacientes vulneráveis e que precisem de um atendimento mais completo voltado para os transtornos mentais.

\section{MÉTODOS}

De início, foi realizada a construção do projeto de pesquisa que foi submetido ao Comitê de Ética em Pesquisa do Centro de Ciências Médicas da Universidade Federal da Paraíba (UFPB) vinculado ao hospital, que recebeu o projeto de pesquisa em 17 de abril de 2018 e o aprovou em sua primeira versão. O CAAE de identificação é 87869618.2.0000.8069.

Para a realização deste estudo, foram escolhidos, em uma amostragem não probabilística por quotas, pacientes acompanhados ambulatorialmente em três especialidades (endocrinologia, cardiologia e reumatologia), sendo estes escolhidas pela carga comórbida de depressão com doenças cardiovasculares, endocrinológicas e dores crônicas?. Como critério de exclusão, foram considerados o preenchimento incorreto ou incompleto dos questionários aplicados e a incapacidade de entendimento dos enunciados e dos quesitos a serem respondidos.

A pesquisa foi realizada no setor ambulatorial do Hospital Universitário Lauro Wanderley da UFPB, localizado na cidade de João Pessoa, no período de $1^{\circ}$ de maio a 30 de junho de 2018. Após o cálculo amostral, que levou em consideração o número de atendimentos anual desses três ambulatórios como universo amostral, chegou-se a um número mínimo de 96 participantes a ser atingido. Foram abordados 109 pacientes, porém oito tiveram preenchimento inacabado pelas demandas de seus atendimentos e, por fim, 101 pacientes foram devidamente entrevistados.

Após a concordância em participar da pesquisa e a assinatura do termo de consentimento livre e esclarecido, os participantes preencheram o questionário sociodemográfico e a escala de depressão de Hamilton.

O primeiro era composto por perguntas sobre identificação pessoal, classificação socioeconômica e uma seção composta por perguntas relacionadas a qualidade do sono, hábitos e estilo de vida, e outras questões sobre a história da doença atual, assim como de antecedentes patológicos relacionados a transtornos mentais.

Já a escala de avaliação de depressão de Hamilton é uma escala traduzida e validada que tem por finalidade avaliar a presença de sintomas depressivos abordando alguns fatores como queixas somáticas, distúrbios do sono, agitação/ retardo e melancolia. A versão utilizada dessa escala é a mais amplamente utilizada, composta por 17 itens $^{8}$.

\section{RESULTADOS}

Os pacientes que compõem a amostra são, predominantemente, procedentes do interior do estado da Paraíba e se definiram como heterossexuais. Estão divididos em 72 (71,3\%) do sexo feminino e 29 (28,7\%) do sexo masculino. Em relação à idade, tem-se que $80 \%$ têm idade compreendida entre $40 \mathrm{e}$ 70 anos. A maioria é composta por pacientes casados (68\%). Noventa e seis pacientes (95\%) afirmaram ter algum tipo de religiosidade, sendo 68 (71\%) católicos, 25 (26\%) evangélicos, 1 (1\%) adventista e 2 (2\%) testemunhas de Jeová.

Em relação à ocupação dos respondentes, tem-se que 27 (27\%) deles são aposentados, 34 (33\%) são autônomos, 20 (20\%) são funcionários públicos, 4 (4\%) são estudantes e 16 (16\%) estão desempregados (do lar). Em relação à classificação socioeconômica, a maior parte da amostra está estratificada na classe C, somando 53,6\% dos indivíduos (29,8\% na classe C1 e 23,8\% na classe C2). Por fim, sobre a questão relacionada a convívio/moradia, foi visto que a maior parcela - $72(71 \%)$ - reside com os cônjuges. Foram avaliados 43 (42\%) pacientes atendidos nos ambulatórios de cardiologia, 23 (23\%), de endocrinologia e 35 (35\%), de reumatologia.

Dos pacientes em estudo, 20 (19,8\%) afirmaram ser sua primeira consulta; dentre os que já estavam em acompanhamento, 32 (31,7\%) fazem tratamento cardiológico (entre os diagnósticos, o mais citado foi hipertensão arterial sistêmica, em 35\% dos casos), 21 (20,8\%) fazem tratamento reumatológico (entre os diagnósticos, o mais citado foi artrite reumatoide, em 39\% dos casos) e 28 (27,7\%) fazem tratamento endocrinológico (entre os diagnósticos, os mais citados foram diabetes mellitus tipo II e hipotireoidismo, em aproximadamente $49 \%$ dos casos).

Em relação às atividades físicas, 35 (34,6\%) desses pacientes afirmam praticar em média três vezes por semana. E por fim, 20 (19,8\%) pacientes relataram que fizeram uso de alguma substância psicoativa nos últimos 30 dias; entre eles, 13 (65\%) fizeram uso de álcool e 7 (35\%), de cigarros. Todos negaram o consumo de drogas ilícitas.

Em relação à qualidade do sono, 38 (37\%) dos pacientes consideram o sono de boa qualidade, 36 (36\%), regular e 27 (27\%), ruim, de acordo com o gráfico a seguir. Do total de respondentes, 54 (53,5\%) já fizeram uso de medicamentos, em específico, para dormir. Eles se encontram distribuídos de acordo com a tabela 1.

Tabela 1. Distribuição do uso de medicamentos para dormir

\begin{tabular}{lcc}
\hline Medicação & $\mathbf{n}$ & $\%$ \\
\hline Alprazolam & 6 & $5,9 \%$ \\
Amitriptilina & 2 & $2,0 \%$ \\
Clonazepam & 15 & $14,8 \%$ \\
Diazepam & 7 & $7,0 \%$ \\
Medicação fitoterápica & 4 & $3,9 \%$ \\
Zolpidem & 3 & $2,9 \%$ \\
Outros/não lembra & 17 & $16,8 \%$
\end{tabular}


Tanto os pacientes que fazem uso de medicamento quanto os que não o fazem relatam um tempo de espera até dormir que varia de 5 minutos até mais de 3 horas. Todavia, os pacientes que utilizam tais medicamentos esperam, em média, 60 minutos até adormecer, enquanto os pacientes que não utilizam esperam, em média, cerca de 45 minutos até dormir. De modo geral, dos 101 pacientes inclusos neste estudo, foi observado que 73 (72,3\%) deles costumam acordar à noite, enquanto 28 (27,7\%) não relatam a ocorrência desse hábito.

Foi observado que $26(25,7 \%)$ pacientes já fizeram tratamento psiquiátrico, e cinco indivíduos (4,9\%) ainda o fazem atualmente. Dos pacientes, 30 (29,7\%) afirmam que fazem uso de psicotrópicos, distribuídos de acordo com a tabela 2. E em caso de necessidade, 98 (97,1\%) afirmam que fariam uma consulta com um psiquiatra.

Utilizando-se os escores averiguados, 10 (9,9\%) dos participantes da pesquisa foram classificados como normais, 29 (28,7\%), como limítrofes, 33 (32,7\%), como ligeiramente deprimidos, 23 (22,8\%), como moderadamente deprimidos e 6 $(5,9 \%)$, como gravemente deprimidos.

A seguir, a variável do desfecho do estudo (sintomas depressivos) foi dividida em duas classificações: 62 pacientes estavam ligeiramente a gravemente deprimidos $(61,4 \%)$, e 39 pacientes não apresentavam essa característica (38,6\%). Por meio do teste qui-quadrado, foi verificado que existe associação significativa entre pacientes ligeiramente a gravemente deprimidos e as seguintes variáveis: "prática de atividade física", "uso de medicamento para dormir", "fazer algum tipo de tratamento paralelo", "tratamento psiquiátrico atualmente", "tratamento psiquiátrico anteriormente" e "uso de psicotrópicos".

De acordo com os p-valores do teste qui-quadrado, listados na tabela 3, observa-se significativa associação entre o fato de o paciente se classificar como ligeiramente a gravemente deprimido com a falta de prática de atividade física, o fato de já fazer algum tipo de tratamento de saúde (não ser a primeira consulta) e o fato de ter feito tratamento psiquiátrico anteriormente.

Tabela 2. Distribuição de psicotrópicos

\begin{tabular}{llc}
\hline Medicamentos & N & $\%$ \\
\hline Alprazolam & 4 & $13,3 \%$ \\
Amitriptilina & 2 & $6,7 \%$ \\
Clonazepam & 4 & $13,3 \%$ \\
Diazepam & 5 & $16,7 \%$ \\
Duloxetina & 2 & $6,7 \%$ \\
Escitalopram & 2 & $6,7 \%$ \\
Sertralina & 3 & $10,0 \%$ \\
Zolpidem & 2 & $6,7 \%$ \\
Outros & 6 & $16,8 \%$ \\
\hline
\end{tabular}

Ao se estudar em qual das especialidades houve maior quantidade de pacientes classificados como ligeiramente a gravemente deprimidos, foi visto que 18 (78\%) dos 23 pacientes de reumatologia obtiveram essa classificação, além de 27 (63\%) dos 43 pacientes de cardiologia e 17 (48\%) dos 35 pacientes de endocrinologia.

Em relação à medicalização para dormir, foi visto que 27 (63\%) dos 43 pacientes de cardiologia, 14 (61\%) dos 23 pacientes de reumatologia e 13 (37\%) dos 35 pacientes de endocrinologia relataram o consumo regular.

De acordo com a verificação de outras associações, foi constatado que, considerando as características de sintomas somáticos, o peso nos membros, nas costas ou na cabeça, além de dores nas costas, cefaleia, mialgia, perda de energia e cansaço, também são características que estão associadas aos pacientes levemente a gravemente deprimidos, apresentando a estatística $\times 2=0,148$, com $p$-valor $=0,14$.

Em relação aos 27 pacientes que declaram qualidade do sono ruim, foi constatado que: 21 (78\%) deles fazem uso de medicamentos para dormir; 25 (92\%) deles acordam durante a noite; a maioria deles (60\%) não pratica atividade física; a maioria deles (63\%) faz uso de psicotrópicos; $81 \%$ deles estão dentro da classificação de levemente a gravemente deprimidos. Por fim, em relação aos pacientes normais e limítrofes, foi visto que apenas 5 (13\%) deles fazem uso de psicotrópicos.

\section{DISCUSSÃO}

De acordo com os resultados desta pesquisa, percebe-se maior prevalência de mulheres buscando os serviços de saúde, principalmente entre 40 e 70 anos. A maioria dos entrevistados é de pessoas casadas, que moram com os seus cônjuges e que possuem algum tipo de religiosidade.

De maneira geral, as mulheres buscam mais esses serviços do que os homens, algo que pode estar relacionado a variações de necessidades entre os gêneros, com maior foco feminino em promoção e prevenção da saúde, bem como a procura de serviços de saúde quando necessário?.

Tabela 3. Associação entre pacientes com algum grau de depressão e outras variáveis

\begin{tabular}{lccc}
\hline \multirow{2}{*}{ Pacientes ligeiramente a gravemente deprimidos } & \multicolumn{3}{c}{ Teste qui-quadrado } \\
\cline { 2 - 4 } & QQ & GL & valor dep \\
\hline Prática de atividade física & 0,043 & 1 & 0,8349 \\
Uso de medicamento para dormir & 10,350 & 1 & 0,0025 \\
Ocorrência de tratamento paralelo & 1,364 & 1 & 0,2409 \\
Tratamento psiquiátrico atualmente & 4,731 & 1 & 0,0296 \\
Tratamento psiquiátrico anteriormente & 0,909 & 1 & 0,3404 \\
Uso de psicotrópicos & 8,6720 & 1 & 0,0032 \\
\hline
\end{tabular}


Nos achados de Gonçalves et. al. ${ }^{9}$, foi observado que ser casado, viver com o companheiro e ter apoio social possui efeito protetor contra a depressão, o que não foi confirmado em nosso estudo. Vimos que a maioria dos entrevistados possuía algum tipo de ocupação e era de classe socioeconômica $C$, segundo os critérios da Associação Brasileira de Empresas de Pesquisa, divulgados em 2016. De acordo com Hämmig e Bauer ${ }^{10}$, o trabalho interfere de maneira direta na saúde mental do indivíduo, já que ele é desenvolvido na rotina diária dele. Nesse prisma, ambientes estressantes podem gerar sintomas negativos, como ansiosos e depressivos.

Rondina et al. ${ }^{11}$ afirmam que o alcoolismo e o tabagismo costumam estar relacionados a sintomas depressivos. Os achados são corroborados pelo estudo em questão, com o uso de substância psicoativa em 19,8\% dos entrevistados, sendo o álcool em 65\% e o cigarro em 15\%.

Em 27\% dos entrevistados, o sono foi classificado como ruim, tendo 53,3\% da amostra usado algum medicamento para dormir. No Brasil, o diazepam foi um dos três princípios ativos mais utilizados entre 2007 e 2010², o que embasa o achado da maior incidência de uso dessa droga pelos entrevistados com a finalidade de melhorias na qualidade do sono, sendo a prescrição para ansiedade e depressão as mais frequentes ${ }^{13}$. A alta frequência de utilização desses medicamentos, principalmente benzodiazepínicos, torna-se preocupante pelos efeitos adversos e de dependência dessas classes, como foi defendido por Charlson et al. ${ }^{14}$, que ainda relacionaram seus malefícios a dosagem, duração do tratamento e potência de cada medicamento.

Em um estudo recente, Minaya et al. ${ }^{15}$ sugerem que o uso de benzodiazepínicos para o tratamento de insônia, por exemplo, não deve ultrapassar um período maior do que quatro semanas, enquanto Fernández García et al. ${ }^{16}$ recomendam no máximo dois meses. Muitos dos entrevistados da nossa pesquisa faziam uso há mais de um ano sem acompanhamento médico regular e ainda se constatou um maior tempo para o início do sono do que naqueles que não usavam, o que reafirma a necessidade de seguimento médico cuidadoso e do estabelecimento de tempo-limite, já que essa classe gera dependência e tolerância.

Mendes et al. ${ }^{17}$ constataram que cardiologistas prescrevem benzodiazepínicos rotineiramente com a justificativa de alívio de sintomas e queixas e manutenção de prescrições anteriores, sendo em mais de 50\% dos casos a pedido de pacientes, parentes e amigos. Ao comparar as três especialidades deste estudo, a cardiologia foi a que mais apresentou pacientes em uso de medicamento para dormir (63\%), sendo mais da metade desses benzodiazepínicos, o que é corroborado pela literatura. Pelos seus malefícios ${ }^{14}$, é necessária maior cautela por parte dos médicos, seja qual for a especialidade.

Mello et al. ${ }^{18}$ defendem que o sono de pessoas que realizam atividade física é melhor do que o de sedentárias e afirmam que o exercício físico melhora a qualidade do sono, principalmente em pessoas inativas. Esse dado é corroborado pela associação de que $60 \%$ das pessoas que relataram ter sono ruim não realizam atividade física. Segundo um estudo epidemiológico realizado em São Paulo, 28,9\% das pessoas fisicamente ativas relataram insônia contra 71,1\% que relataram sonolência excessiva ${ }^{19}$.

Um estudo de coorte realizado entre 1999 e 2010 verificou a incidência inversamente proporcional entre a prática de exercícios físicos e sintomas depressivos ${ }^{20}$. Já uma metanálise realizada com artigos que datavam até 2017 em cinco base de dados diferentes atestou que exercícios de resistência diminuem significativamente sintomas depressivos entre adultos, independentemente da situação de saúde ${ }^{21}$. Além disso, observou-se que a atividade física pode, de fato, ser uma estratégia na redução do risco para o desenvolvimento da depressão ${ }^{22}$.

Vimos que apenas 4,9\% dos pacientes do estudo faziam tratamento psiquiátrico atualmente, e 61,4\% deles foram classificados, de acordo com Furukawa et al. ${ }^{23}$, como ligeiramente a gravemente deprimidos. De fato, é bastante difícil o reconhecimento da depressão em pacientes clínicos, seja pela abordagem médica, seja pela falta de verbalização dos sintomas por eles ${ }^{6}$. Seja pelo tempo limitado, seja pela falta de treinamento e de conhecimento anterior do indivíduo, a depressão pode não ser reconhecida nos pacientes clínicos. Os médicos, por sua vez, consideram normal qualquer manifestação de sofrimento devido às condições clínicas dos pacientes $^{6}$. A depressão, muitas vezes, não é diagnosticada e tratada em ambulatórios de outras especialidades quando o paciente procura o serviço por acometimentos físicos. Habitualmente, as queixas do paciente se associam a acometimentos diversos e mal-estar indefinido, o que se apresenta com um fator de maior dificuldade no diagnóstico ${ }^{7}$.

A hipertensão arterial sistêmica mostra-se com importante fator de risco no desenvolvimento de doenças cardíacas, como doença arterial coronariana. A morbimortalidade cardíaca é mais intensa em pacientes deprimidos ${ }^{24}$. Sendo assim, é preciso cuidar do paciente como um todo, e não apenas da sua condição cardíaca. No estudo, encontrou-se que a doença mais prevalente no ambulatório de cardiologia foi a hipertensão, o que indica a necessidade de um olhar global para o paciente que entra no consultório, já que sua condição psíquica implica pior prognóstico.

Referente à endocrinologia, percebeu-se uma maior quantidade de pacientes com diabetes mellitus, obesidade e hipotireoidismo. A prevalência de transtornos de humor em diabéticos, por exemplo, alcança 53,6\%. Considera-se que a conexão entre obesidade e depressão seja bidirecional, sendo o peso afetado pelo o humor e o humor afetado indiretamente pelo peso. Já o hipotireoidismo frequentemente se associa a sintomas depressivos e ansiosos, incluindo diminuição do apetite, fadiga, retardo psicomotor e letargia ${ }^{4}$.

Por fim, a associação entre doenças reumatológicas e transtornos psiquiátricos tem alta prevalência. As principais 
comorbidades psiquiátricas em pacientes com artrite reumatoide, que foi a mais presente nos entrevistados, são transtornos de ansiedade e transtornos depressivos. A prevalência de pacientes depressivos com a condição reumática citada, de acordo com a literatura, é de 13\% a 20\% e a de pacientes com transtornos ansiosos é de 8,8\% a 13\%4.

\section{CONCLUSÃO}

Existe uma alta prevalência de sintomas depressivos em pacientes dos ambulatórios de clínica médica, com destaque para as doenças cardiovasculares, endocrinológicas e reumatológicas. Percebe-se que houve um grande destaque para os sintomas somáticos, o que pode explicar a grande quantidade de entrevistados que não são acompanhados por psiquiatra e são tratados apenas em relação à doença física.

Os médicos são formados de forma lacunar e, muitas vezes, não conseguem analisar o paciente como o todo e só suspeitam de depressão quando há nesses pacientes sintomas como tristeza e melancolia. Isso pode estar acontecendo devido à falta de comunicação que há entre psiquiatras e especialistas de outras áreas, assim como por um preparo ineficaz em identificar esses sintomas que vem desde a formação.

É preciso haver maior comunicação entre os profissionais da área médica, como também o desenvolvimento de ferramentas que os ajudem a identificar sintomas depressivos quando muito deles forem confusos ou inconclusivos. Tanto a aplicação de escalas, como foi feita nesses estudos, quanto uma simples triagem são exemplos de alternativas que poderiam ser utilizadas com a intenção de diminuir a quantidade de pacientes subdiagnosticados e tratados de forma inadequada.

Para isso, é necessária a criação de ambulatórios multiprofissionais, compostos de psiquiatras e psicólogos, a fim de encurtar essa distância e diminuir os estigmas ainda existentes referentes ao adoecimento mental. A otimização dos serviços de referência e contrarreferência, desburocratizando-o e facilitando o manejo clinico dos pacientes, bem como um maior investimento no setor ambulatorial da instituição, é essencial.

\section{CONTRIBUIÇÕES INDIVIDUAIS}

Todos os autores contribuíram significativamente na concepção e desenho do estudo, análise, interpretação dos dados, bem como na elaboração do artigo e na aprovação da versão final a ser publicada.

\section{CONFLITO DE INTERESSES}

Nenhum dos autores apresentou divergência de interesses ao longo da elaboração do projeto e execução do trabalho.

\section{REFERÊNCIAS}

1. Botega NJ. Prática psiquiátrica no hospital geral: interconsulta e emergência. $2^{a}$ ed. São Paulo: Artmed; 2006

2. Andrade LH, Wang YP, Andreoni S, Silveira CM, Alexandrino-Silva C, Siu ER, et al. Mental Disorders in Megacities: Findings from the São Paulo Megacity Mental Health Survey, Brazil. PLoS One. 2012;7(2):e31879.

3. Organização Panamericana de Saúde; Organização Mundial da Saúde. Com depressão no topo da lista de causas de problemas de saúde, OMS lança a campanha "Vamos conversar". Disponível em: http://www.paho.org/bra/index.php?option=com_ content\&viem=article\&id=5385: com-depressa0-no-topo-da-lista-de-problemas-desaude-oms-lanca-a-campanha-vamos-conversar\&ltemid=839. Acesso em: 26 abr. 2018.

4. Humes EC, Vieira MEB, Fráguas Jr. R. Psiquiatria Interdisciplinar. Barueri, SP: Manole; 2016.

5. Lima P. Rastreamento de transtornos psiquiátricos em pacientes ambulatoriais atendidos em um hospital oncológico [dissertação]. Barretos: Fundação Pio XIl; 2014.

6. Furlanetto LM, Brasil MA. Diagnosticando e tratando depressão no paciente com doença clínica. J Bras Psiquiatr. 2006;55(1):8-19.

7. Teng CT, Humes EC, Demetrio FN. Depressão e comorbidades clínicas. Rev Psiquiatr Clin. 2005;32(3):149-59.

8. Carvalho FR, Lima MG, Azevedo RCG, Caetano D. Tradução do inglês para o português do questionário de autoavaliação da Escala de Hamilton para a depressão. J Bras Psiquiatr. 1993:42(5):255-60.

9. Gonçalves AMC, Teixeira MTB, Gama JRA, Lopes CS, Silva GA, Gamarra CJ, et al. Prevalência de depressão e fatores associados em mulheres atendidas pela Estratégia de Saúde da Família. J Bras Psiquiatr. 2018;67(2):101-9.

10. Hämmig 0, Bauer GF. Work, work-life conflict and health in an industrial work environment. Occup Med (Lond). 2014;64(1):34-8.

11. Rondina RC, Gorayeb R, Botelho C. Características psicológicas associadas ao comportamento de fumar tabaco. J Bras Pneumol. 2007;33(5):592-601

12. Agência Nacional de Vigilância Sanitária. Panorama dos dados do sistema nacional de Gerenciamento de produtos controlados: um sistema para o monitoramento de medicamentos no Brasil. Boletim de Farmacoepidemiologia. 2011. Disponível em: http://www.anvisa.gov. br/sngpc/boletins/2011/boletim_sngpc_2edatualizada.pdf. Acesso em: 7 maio 2018.

13. Alvim MM. Prevalência de uso de benzodiazepínicos em idosos e fatores associados [dissertação]. Juiz de Fora: Universidade Federal de Juiz de Fora; 2016.

14. Charlson F, Degenhardt L, McLaren J, Hall W, Lynskey M. A systematic review of research examining benzodiazepine-related mortality. Pharmacoepidemiol Drug Saf. 2009;18(2):93-103.

15. Minaya 0, Ugalde 0, Fresán A. Uso inapropiado de fármacos de prescripción: dependencia a benzodiazepinas en adultos mayores. Salud Ment. 2009;32(5):405-11.

16. Fernández García A, González Viña A, Penã Machado MA. Bases científicas para el uso de lasbenzodiazepinas. Rev Cubana Med Gen Integr. 2003;19(1).

17. Mendes CMM, Souza FCF, Cunha TBL. Benzodiazepínicos na prescrição de cardiologistas. Revista Norte Nordeste de Cardiologia. 2017;7(3):4-10.

18. Mello TM, Fernandez AC, Tufik S. Levantamento epidemiológico da prática de atividade física na cidade de São Paulo. Rev Bras Med Esporte. 2000;6(4):119-24.

19. Mello MT, Boscolo RA, Esteves AM, Tufik S. 0 exercício físico e os aspectos psicobiológicos. Rev Bras Med Esporte. 2005;11(3):203-7.

20. Willis BL, Leonard D, Barlow CE, Martin SB, DeFina LF, Trivedi MH. Association of Midlife Cardiorespiratory Fitness With Incident Depression and Cardiovascular Death After Depression in Later Life. JAMA Psychiatry. 2018;75(9):911-7.

21. Gordon BR, McDowell CP, Hallgren M, Meyer JD, Lyons M, Herring MP. Association of Effcacy of Resistance Exercise Training With Depressive Symptoms: Meta-analysis and Metaregression Analysis of Randomized Clinical Trials. JAMA Psychiatry. 2018;75(6):566-76.

22. Mammen G, Faulkner G. Physical activity and the prevention of depression: a systematic review of prospective studies. Am J Prev Med. 2013;45(5):649-57.

23. Furukawa TA, Akechi T, Azuma H, Okuyama T, Higuchi T. Evidence-based guidelines for interpretation of the Hamilton Rating Scale for Depression. J Clin Psychopharmol. 2007;27(5):531-3.

24. Lotufo NF, Yacubian J, Scalco AZ, Gonçalves L. Terapia comportamental cognitiva dos transtornos afetivos. In: Rangé B, org. Psicoterapias Cognitivo-Comportamentais: um diálogo com a psiquiatria. Porto Alegre: Artmed; 2001. 